\section{Cutting off growth}

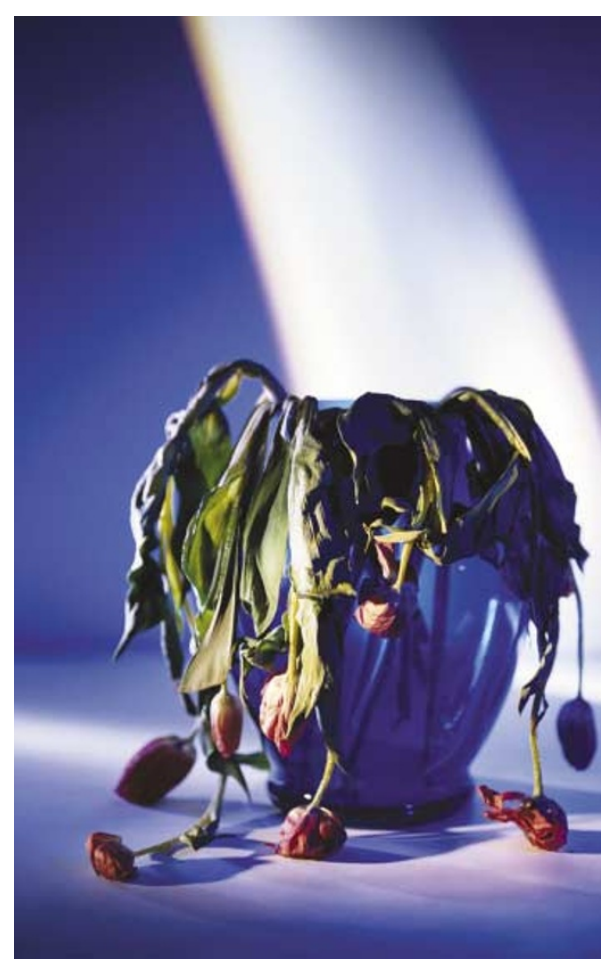

Tumour progression cannot occur without angiogenesis, which supplies the necessary oxygen and nutrients to the growing tumour. In the August issue of Nature Medicine, Levon Khachigian and colleagues describe a technique for cutting off this supply — quite literally — by using DNAbased enzymes (DNAzymes) to chop up early growth response factor-1 (Egr1) mRNA. EGR1 is a transcription factor, which the authors show to be integral in the control of endothelialcell growth and tumour angiogenesis.

The DNAzymes that had been engineered to target human EGR mRNA inhibited endothelial-cell migration in an in vitro assay and inhibited capillary tubule formation by endothelial cells cultured on basement-membrane matrices (Matrigel). Blood-vessel formation within Matrigel plugs that had been injected subcutaneously into mice was also blocked by a mouse Egr 1 DNAzyme (ED5). Use of a control DNAzyme - with a scrambled hybridization sequence - had no effect in any of these experiments. Med. 9, 1026-1032 (2003)
ED5 can therefore inhibit angiogenesis, but what effect does this inhibition have on tumour growth? When ED5 was administered subcutaneously to $\mathrm{BALB} / \mathrm{c}$ nude mice that had been injected with MCF-7 breast cancer cells, a fivefold reduction in tumour size was seen compared with mice injected with a scrambled DNAzyme or vehicle alone. ED5 had no direct inhibitory effect on MCF-7 proliferation; it decreased vascular density in the tumours. Local intratumoral injection also blocked MCF-7 tumour growth. Importantly, no systemic side effects, on wound healing, haemostasis or reproduction were seen.

So, how does inhibition of Egr 1 block angiogenesis? The authors looked at the effect of Egr 1 inhibition on two potent inducers of angiogenesis - fibroblast growth factor-2 (Fgf2) and vascular endothelial growth factor (Vegf). Expression of Egr1 and Fgf2 was weak or not present in the endothelium of the ED5-treated mouse MCF-7 tumours, whereas Vegf expression was unchanged on treatment with ED5. The DNAzyme had no effect on the growth of B16 malignant melanoma in mice B16 is dependent on the Vegf pathway, but not on Fgf2, for growth and metastasis.

EGR1 therefore has a crucial role in endothelial-cell growth and tumour angiogenesis and this seems to be regulated by FGF2 and not VEGF. It might be interesting to investigate DNAzymes, such as those targeting EGR1, as a new class of anticancer agents.

Ezzie Hutchinson

\section{(2) References and links} ORIGINAL RESEARCH PAPER Fahmy, R. G. et al. Transcription factor Egr-1 supports FGFdependent angiogenesis during neovascularization and tumour growth. Nature WEB SITE

Levon Khachigian's lab:

Levon Khachigian's lab: /8571226?:OpenDocument

\section{IN BRIEF}

\section{METASTASIS}

Transforming growth factor- $\beta$ signaling impairs Neuinduced mammary tumorigenesis while promoting pulmonary metastasis.

Siegel P. M. et al. Proc. Natl Acad. Sci. USA 100, 8430-8435 (2003)

There is evidence that primary tumour cells can reprogramme their response to TGF- $\beta$, turning this tumour suppressor into a metastasis-inducing factor. Siegel et al. used transgenic mouse models to show that expression of the activated NEU receptor tyrosine kinase (also known as ERBB2/HER2) plus the activated type I TGF- $\beta$ receptor suppressed mammary tumour growth, promoting pulmonary metastasis by specifically enhancing extravasation of cancer cells, while impairing tumour growth.

\section{TUMORIGENESIS}

The ErbB2/ErbB3 heterodimer functions as an oncogenic unit: ErbB2 requires ErbB3 to drive breast tumor cell proliferation.

Holbro, T. et al. Proc. Natt Acad. Sci. USA 100, 8933-8938 (2003)

For ERBB2 to function as a receptor tyrosine kinase, it must dimerize with another member of the ERBB family. As ERBB2expressing tumours co-express ERBB3, Holbro et al. investigated whether ERBB3 is required for transformation. Suppressing ERBB3 expression in ERBB2-overexpressing breast cancer cells blocked proliferation, which could be restored by expression of constitutively active protein-kinase B. Targeted disruption of this heterodimer could prove useful for the treatment of breast cancer.

\section{TUMOUR SUPPRESSORS}

\section{p53- and Mdm2-independent repression of NFkB} transactivation by the ARF tumor suppressor.

Rocha, S., Campbell, K. J. \& Perkins, N. D. Mol. Cell 12, 15-25 (2003)

Activity of the NFKB family of transcription factors, particularly RELA, is associated with tumorigenesis and represses p53 function. Rocha et al. predicted that ARF might alter RELA activity to improve its function as a tumour suppressor. They showed that ARF causes RELA to associate with the histone deacetylase HDAC1, which represses transcription of RELA. More importantly, ARF represses the transcriptional activity of RELA in response to the oncogene $B C R-A B L$.

\section{METASTASIS}

Sphingosylphosphorylcholine regulates keratin network architecture and visco-elastic properties of human cancer cells.

Michael Beil et al. Nature Cell Biol. 24 Aug 2003 (doi: 10.1038ncb1037)

Sphingosylphosphorylcholine (SPC) is a biologically active lipid that is found at increased concentrations in the blood and malignant ascites of patients with ovarian cancer. The authors show that SPC reorganizes keratin filaments, which increases the elasticity of cells and therefore aids their migration. So, SPC might facilitate migration of cells during metastasis. 